\title{
Effective-Range Approach and Scaling Laws for Electromagnetic Strength in Neutron-Halo Nuclei
}

\author{
S. Typel \\ Gesellschaft für Schwerionenforschung mbH (GSI), Planckstraße 1, D-64291 Darmstadt, Germany
}

G. Baur

Institut für Kernphysik, Forschungszentrum Jülich, D-52425 Jülich, Germany

(Received 22 June 2004; published 29 September 2004)

\begin{abstract}
We study low-lying multipole strength in neutron-halo nuclei. The strength depends only on a few low-energy constants: the neutron separation energy, the asymptotic normalization coefficient of the bound-state wave function, and the scattering length that contains the information on the interaction in the continuum. The shape of the transition probability shows a characteristic dependence on few scaling parameters and the angular momenta. The total $E 1$ strength is related to the root-mean-square radius of the neutron wave function in the ground state and shows corresponding scaling properties. We apply our approach to the $E 1$ strength distribution of ${ }^{11} \mathrm{Be}$.
\end{abstract}

DOI: 10.1103/PhysRevLett.93.142502

Electromagnetic dipole strength in stable nuclei is concentrated in the giant dipole resonance. In recent years it was found that there is an appreciable low-lying $E 1$ strength in light neutron-rich nuclei $[1,2]$. This phenomenon is especially pronounced in neutron-halo nuclei, where it can be explained essentially as a singleparticle effect [3,4]. Examples are ${ }^{11} \mathrm{Be}[5,6]$ and ${ }^{19} \mathrm{C}[7]$. This low-lying strength was observed mainly in Coulomb dissociation experiments; for a recent review, see [8].

The dissociation cross section directly reflects the dipole strength distribution or reduced transition probability $d B(E 1) / d E$. Photodissociation of and radiative capture leading to halo nuclei provide equivalent information. The measured strength distributions show simple features. They tend to be universal when plotted in the appropriate reduced parameters [9] because low-energy processes do not depend on certain details of the interaction. Consequently, effective field theories are nowadays used for the description of halo nuclei [10]. In Ref. [11] nonrelativistic two-body scattering by a short range potential was studied using the renormalization group. It was found that the expansion around the nontrivial fixed point is equivalent to the effective-range (ER) expansion.

It is the purpose of this Letter to apply the ER approach to one-neutron-halo nuclei and find scaling laws for the transition strength. This work is similar in spirit to effective field theories: the effects of unknown short distance behavior are parametrized by a few low-energy constants. It is not our aim to relate these parameters to the many-body physics.

General expressions for electromagnetic strength distributions in halo nuclei were derived before (see, e.g., [12], and references therein, for proton + core systems), however, often neglecting the nuclear continuum interaction. Also expansions for small relative energies were obtained [13]. The application of ER theory to the study of halo nuclei was considered before, e.g., in the descrip-
PACS numbers: $24.50 .+\mathrm{g}, 25.20 .-\mathrm{x}, 25.60 . \mathrm{Gc}, 27.20 .+\mathrm{n}$

tion of ground state properties [14], and for radiative capture cross sections into $s$-, $p$-, and $d$-wave bound states taking into account the interaction only for $s$-waves assuming a zero-range potential [15]. The effect of the interaction in the continuum states on direct neutron capture and photodisintegration of ${ }^{13} \mathrm{C}$ was studied in $[16,17]$. While they find a sensitivity on neutron optical model parameters for $s \rightarrow p$ capture, this sensitivity is strongly reduced for the cases of $p \rightarrow s$ and $p \rightarrow d$ capture.

The prototype of a neutron-halo nucleus is the deuteron. Radiative capture or photodissociation at low energies are well described by the binding energy and the effective range; see, e.g., [18]. Quite similar, the low-lying $E 1$ strength in neutron-halo nuclei is determined by two parameters if the continuum interaction is neglected: one is the binding energy (or neutron separation energy $S_{n}$ ) which determines the overall shape of the $d B(E 1) / d E$ distribution; the other one is a normalization constant. In the analogous case of ${ }^{19} \mathrm{C}$, the binding energy could be determined directly from the shape of the observed strength distribution [7]. Effects of the neutron-core interaction are parametrized efficiently by the ER approximation.

The cross section for photodissociation of a nucleus $a$ into a neutron $n$ and a core $c$ is given by

$$
\begin{aligned}
\sigma_{\pi \lambda}(a+\gamma & \rightarrow n+c) \\
& =\frac{\lambda+1}{\lambda} \frac{(2 \pi)^{3}}{[(2 \lambda+1) ! !]^{2}}\left(\frac{E_{\gamma}}{\hbar c}\right)^{2 \lambda-1} \frac{d B(\pi \lambda)}{d E} .
\end{aligned}
$$

The reduced transition probability for electromagnetic multipolarity $\pi \lambda$ is denoted by $d B(\pi \lambda) / d E$. The photon energy is given by $E_{\gamma}=E+S_{n}$ with $S_{n}=\hbar^{2} q^{2} /(2 \mu)$, where $q$ is the inverse decay length of the bound-state wave function and $\mu$ is the reduced mass. Using detailed 
balance, the corresponding radiative capture cross section can be obtained from this expression. With the equivalent photon number, cross section (1) determines the (first order) Coulomb dissociation cross section [8].

The strength distribution $d B\left(E \lambda, l_{i} \rightarrow l_{f}\right) / d E$ for a certain transition depends on the corresponding matrix element that contains a radial integral with the wave functions of the initial and final states with orbital angular momenta $l_{i}$ and $l_{f}$, respectively. (We consider spinless neutrons and cores; a generalization is obvious.) At low energies $E=\hbar^{2} k^{2} /(2 \mu)$, the main contribution to the radial integral arises from radii larger than the radius $R$ of the nucleus. This is true for all possible values of the angular momenta involved. In the figures of Refs. [16,17] it can very well be seen that the radial integrals are dominated by the outside region. Away from a resonance, a case which we always assume here, the continuum wave function is small inside the nuclear radius. In a hard sphere model it is exactly zero.

For the neutron + core case the dimensionless reduced radial integral is well approximated by

$$
\begin{aligned}
I_{l_{i}}^{l_{f}}(\lambda)= & -i^{l_{i}} k q^{\lambda+2} \int_{R}^{\infty} d r r^{\lambda+2} h_{l_{i}}^{(1)}(i q r)\left[\cos \left(\delta_{l_{f}}\right) j_{l_{f}}(k r)\right. \\
& \left.-\sin \left(\delta_{l_{f}}\right) y_{l_{f}}(k r)\right]
\end{aligned}
$$

with spherical Bessel $\left(j_{l}\right)$, Neumann $\left(y_{l}\right)$, and Hankel $\left(h_{l}^{(1)}\right)$ functions, respectively, that describe the behavior of the radial wave functions beyond the range of the nuclear potential. The phase shift $\delta_{l_{f}}$ contains the information on the interaction in the final continuum state. Introducing the dimensionless shape functions

$$
S_{l_{i}}^{l_{f}}(\lambda)=\frac{q}{k}\left|I_{l_{i}}^{l_{f}}(\lambda)\right|^{2},
$$

the reduced transition probability is given by

$$
\begin{aligned}
\frac{d B}{d E}\left(E \lambda, l_{i}\right. & \left.\rightarrow l_{f}\right) \\
& =\left[Z_{\mathrm{eff}}^{(\lambda)} e\right]^{2} \frac{2 \mu}{\pi \hbar^{2}} \frac{2 \lambda+1}{4 \pi}\left(l_{i} 0 \lambda 0 \mid l_{f} 0\right)^{2} \frac{\left|C_{l_{i}}\right|^{2}}{q^{2 \lambda+3}} S_{l_{i}}^{l_{f}}(\lambda)
\end{aligned}
$$

with the effective charge number $Z_{\text {eff }}^{(\lambda)}=Z_{c}\left[m_{n} /\left(m_{n}+\right.\right.$ $\left.\left.m_{c}\right)\right]^{\lambda}$ and the asymptotic normalization constant (ANC) $C_{l_{i}}$ of the bound-state wave function. For neutron + core systems the dipole transitions are dominant since $E 2$ transitions are suppressed by the small effective charge.

The ANC of the actual many-body wave function as it appears in (4) consists of the ANC of a neutron singleparticle wave function and a spectroscopic factor which accounts for the many-body aspects. The single-particle ANC is determined by the normalization of the wave function. It depends on $q$ with the scaling behavior $\left|C_{l_{i}}\right|^{2} \propto q$ for $l_{i}=0$ and $\left|C_{l_{i}}\right|^{2} \propto q \gamma^{2 l_{i}-1}$ for $l_{i}>0$ as seen, e.g., in a square-well model. Here, we have introduced the dimensionless scaling parameter $\gamma=q R$. The factor $\left|C_{l_{i}}\right|^{2} / q^{2 \lambda+3}$ in the reduced transition probability (4) depends only on properties of the ground state. It directly shows the scaling with the characteristic parameter $q$. Clearly, for small separation energies of the neutron a large transition strength can be expected.

The reduced radial integrals (2) have the general form

$$
\begin{aligned}
I_{l_{i}}^{l_{f}}(\lambda)= & \frac{\gamma \exp (-\gamma)}{\left(\gamma^{2}+\kappa^{2}\right)^{\lambda+1}}\left(\frac{\gamma}{\kappa}\right)^{l_{f}}\left[\mathcal{R}_{l_{i}}^{(+) l_{f}}(\lambda) \cos \left(\kappa+\delta_{l_{f}}\right)\right. \\
& \left.+\mathcal{R}_{l_{i}}^{(-) l_{f}}(\lambda) \sin \left(\kappa+\delta_{l_{f}}\right)\right]
\end{aligned}
$$

with $\kappa=k R$, where the rational functions $\mathcal{R}_{l_{i}}^{( \pm) l_{f}}(\lambda)$ can be found for all relevant values of $l_{i}$ and $l_{f}$ by means of the recursion relations

$$
\begin{aligned}
\mathcal{R}_{l_{i}}^{( \pm) l_{f}+1}(\lambda+1)= & {\left[2 \kappa^{2}(\lambda+1)+\left(\gamma^{2}+\kappa^{2}\right)\left(2 l_{f}+1\right)\right] } \\
& \times \mathcal{R}_{l_{i}}^{( \pm) l_{f}}(\lambda)-\kappa\left(\gamma^{2}+\kappa^{2}\right) \\
& \times\left[\frac{d}{d \kappa} \mathcal{R}_{l_{i}}^{( \pm) l_{f}}(\lambda) \pm \mathcal{R}_{l_{i}}^{(\mp) l_{f}}(\lambda)\right], \\
\mathcal{R}_{l_{i}+1}^{( \pm) l_{f}}(\lambda+1)= & {\left[2 \gamma^{2}(\lambda+1)+\left(\gamma^{2}+\kappa^{2}\right)\right.} \\
& \left.\times\left(l_{i}+\lambda+1-l_{f}+\gamma\right)\right] \mathcal{R}_{l_{i}}^{( \pm) l_{f}}(\lambda) \\
& -\gamma\left(\gamma^{2}+\kappa^{2}\right) \frac{d}{d \gamma} \mathcal{R}_{l_{i}}^{( \pm) l_{f}}(\lambda)
\end{aligned}
$$

starting from

$$
\begin{array}{ll}
\mathcal{R}_{0}^{(+) 0}(0)=\kappa, & \mathcal{R}_{0}^{(-) 0}(0)=\gamma, \\
\mathcal{R}_{1}^{(+) 1}(0)=-\kappa, & \mathcal{R}_{1}^{(-) 1}(0)=\left[\kappa^{2}(1+\gamma)+\gamma^{2}\right] / \gamma^{2} .
\end{array}
$$

The radial integral involving the regular scattering wave function shows a $r^{\lambda-l_{i}+l_{f}+1}$ behavior for small values of $r$; thus it can be extended to $R=0$. On the other hand, the radial integral involving the irregular function shows a $r^{\lambda-l_{i}-l_{f}}$ behavior. Thus for $\lambda=1$ we can extend the integral to zero only for $l_{i}=0, l_{f}=1$ and $l_{i}=1, l_{f}=0$ and have convergence. In the other cases, one has to cut off the integral for a finite $R$ value.

For low energies we can use the effective-range approximation $k^{2 l+1} \cot \left(\delta_{l}\right)=-1 / a_{l}+r_{l} k^{2} / 2+\cdots$ for the phase shifts $\delta_{l}$ with the scattering length $a_{l}$ and the effective-range $r_{l}$ in order to take the interaction in the continuum state into account. (Note that $a_{l}$ and $r_{l}$ have the dimension of a length only for $l=0$.) We restrict ourselves to the lowest order term, i.e.,

$$
\tan \left(\delta_{l}\right)=-\left(x c_{l} \gamma\right)^{2 l+1}
$$

with the dimensionless reduced scattering length $c_{l}$ defined by $c_{l}^{2 l+1}=a_{l} / R^{2 l+1}$ and the ratio $x=\kappa / \gamma=k / q=$ $\sqrt{E / S_{n}}$. We assume that this is a reasonable approximation of the phase shift. We further assume that the scattering length has a "natural" value with $c_{l}$ being $O(1)$. For example, for $s$-wave scattering from a hard sphere 
with radius $R$ we have $c_{0}=1$. In exceptional cases, this assumption is not fulfilled, e.g., if there are resonances in the low-lying continuum or subthreshold states. For an $s$-wave halo nucleus bound by a zero-range force the scattering length is given by $a_{0}=1 / q$; i.e., $c_{0}=1 / \gamma$. In such a case the scattering length in the $s$ wave is unnaturally large; it diverges in the halo limit $\gamma \rightarrow 0$.

We can expand the analytical results for $S_{l_{i}}^{l_{f}}(\lambda)$ in terms of the small parameter $\gamma$ and obtain for the most important cases for dipole transitions

$$
\begin{gathered}
S_{0}^{1}(1)=\frac{4 x^{3}}{\left(1+x^{2}\right)^{4}}\left[1-c_{1}^{3}\left(1+3 x^{2}\right) \gamma^{3}+\cdots\right], \\
S_{1}^{0}(1)=\frac{x\left(3+x^{2}\right)^{2}}{\left(1+x^{2}\right)^{4}}\left[1-\frac{4 c_{0}}{3+x^{2}} \gamma+\cdots\right] .
\end{gathered}
$$

The term in front of the square bracket in Eq. (10) is the well-known result without continuum interaction; see, e.g., [5,17]. In general there will be a modification of the shape function. It is getting more and more important for larger binding energies of the halo nucleus, i.e., larger $\gamma$. Transitions with $l_{f}=l_{i}-1$ are more affected by this final-state interaction than transitions with $l_{f}=l_{i}+1$. In the case $s \rightarrow p$ the first correction to the shape function for $\gamma \rightarrow 0$ appears only in the $\gamma^{3}$ term proportional to $c_{1}^{3}$. There are no corrections of lower order in $\gamma$ independent of the final-state interaction. This explains the remarkable uniformity of the characteristic shape for the low-lying $d B(E 1) / d E$ strength in halo nuclei such as ${ }^{11} \mathrm{Be}$ and ${ }^{19} \mathrm{C}$. For the $p \rightarrow s$ transition there is a greater sensitivity to the final-state interaction. It appears already as a correction linear in $\gamma$ and $c_{0}$. This is in accord with the results of Ref. [16].

The total transition strength can be obtained by integrating (4) over the relative energy $E$. It is given by

$B\left(E \lambda, l_{i} \rightarrow l_{f}\right)=\left[Z_{\text {eff }}^{(\lambda)} e\right]^{2} \frac{2 \lambda+1}{4 \pi}\left(l_{i} 0 \lambda 0 \mid l_{f} 0\right)^{2} \frac{\left|C_{l_{i}}\right|^{2}}{q^{2 \lambda+1}} \mathcal{T}_{l_{i}}^{l_{f}}(\lambda)$

with

$$
\mathcal{T}_{l_{i}}^{l_{f}}(\lambda)=\frac{2}{\pi} \int_{0}^{\infty} d x x S_{l_{i}}^{l_{f}}(\lambda)=\frac{2}{\pi} \int_{0}^{\infty} d x\left|I_{l_{i}}^{l_{f}}(\lambda)\right|^{2} .
$$

For dipole transitions and no final-state interaction (i.e., $c_{l}=0$ corresponding to a plane wave in the final state), we find

$$
\begin{gathered}
\mathcal{T}_{1}^{0}(1)=\mathcal{T}_{1}^{2}(1)=\frac{1}{4}\left(5+6 \gamma+2 \gamma^{2}\right) e^{-2 \gamma}, \\
\mathcal{T}_{0}^{1}(1)=\frac{1}{4}\left(1+2 \gamma+2 \gamma^{2}\right) e^{-2 \gamma}, \\
\mathcal{T}_{2}^{1}(1)=\frac{1}{4 \gamma}\left(36+37 \gamma+14 \gamma^{2}+2 \gamma^{3}\right) e^{-2 \gamma} .
\end{gathered}
$$

A finite value of $\gamma$ leads to a reduction of the total strength in the continuum as compared to the extreme halo limit $\gamma=0$. With final-state interaction, the functions $\mathcal{T}_{l_{i}}^{l_{f}}(\lambda)$ also depend on the reduced scattering length $c_{l}$; however, rather lengthy expressions are obtained.

The value of $B\left(E 1, l_{i}\right)=\sum_{l_{f}} B\left(E 1, l_{i} \rightarrow l_{f}\right)$ for all $E 1$ transitions from a state with orbital angular momentum $l_{i}$ is directly related by the non-energy-weighted sum rule

$$
B\left(E 1, l_{i}\right)=\left[Z_{\mathrm{eff}}^{(1)} e\right]^{2} \frac{3}{4 \pi}\left\langle r^{2}\right\rangle_{l_{i}}
$$

to the root-mean-square (rms) radius $\left\langle r^{2}\right\rangle_{l_{i}}$ of the same bound state; see, e.g., [2]. The rms radius scales with $\gamma$ for different values of the bound-state orbital angular momentum $l_{i}$ as $\left\langle r^{2}\right\rangle_{0} \propto R^{2} / \gamma^{2},\left\langle r^{2}\right\rangle_{1} \propto R^{2} / \gamma$, and $\left\langle r^{2}\right\rangle_{l_{i}} \propto R^{2}$ for $l_{i} \geq 2$ [4]. On the other hand, the energy-weighted sum rule (Thomas-Reiche-Kuhn sum rule) gives a constant independent of $l_{i}$. From the above we see clearly that the low-lying strength is most pronounced for $s$-wave bound states and to a lesser degree for $p$-wave bound states in halo nuclei. Considering the $q$ dependence of $B\left(E 1, l_{i} \rightarrow l_{f}\right)$ in (12) the scaling of $\left\langle r^{2}\right\rangle_{l_{i}}$ with $\gamma$ is exactly reproduced. With no final-state interaction the full transition strength as predicted by the sum rule is found in the continuum. However, if there are bound states in the particular final state the continuum contribution to the total strength is reduced and the shape will be distorted. This will correspond to a nonzero value of $c_{l}$.

Let us now apply the general theory to ${ }^{11} \mathrm{Be}$, which shows a very pronounced single-particle halo structure with a neutron bound by $504 \mathrm{keV}$ in the $\frac{1}{2}^{+}$ground state that can be considered as a $2 s_{1 / 2}$ state. Assuming a radius of $R=2.78 \mathrm{fm}$ the neutron separation energy corresponds to a small value $\gamma=0.4132<1$ typical for a halo nucleus. Unlike the case of the deuteron, where there is no excited bound state, there is a $\frac{1}{2}^{-}$bound state at $320 \mathrm{keV}$ excitation energy. We can regard it to a good approximation as a $1 p_{1 / 2}$ single-particle state. We perform a $\chi^{2}$ fit of our analytical ER approach (not using the expansion for small $\gamma$ ) to the experimental Coulomb dissociation data [6] by adjusting the reduced scattering lengths $c_{1}^{3 / 2}$ and $c_{1}^{1 / 2}$ in the two $p$-wave channels (see Table I) and the ground state $\mathrm{ANC} C_{0}$. The quantity $c_{1}^{1 / 2}$ is definitely unnaturally large as expected from the existence of the weakly bound $\frac{1}{2}^{-}$state. In Fig. 1 our model fit

TABLE I. Dimensionless quantities $c_{1}^{j}$, see Eq. (9), and spectroscopic factors $C^{2} S$ from the fit to experimental data [6] of ${ }^{11} \mathrm{Be}$ Coulomb dissociation.

\begin{tabular}{lccc}
\hline \hline Model & $c_{1}^{3 / 2}$ & $c_{1}^{1 / 2}$ & $C^{2} S$ \\
\hline Effective-range & $-0.41(86,-20)$ & $2.77(13,-14)$ & $0.704(15)$ \\
Woods-Saxon & $-0.46(70,-14)^{\mathrm{a}}$ & $1.87(3)^{\mathrm{a}}$ & $0.696(15)$ \\
\hline \hline
\end{tabular}

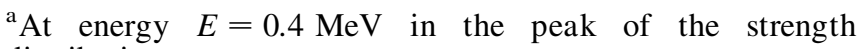
distribution. 


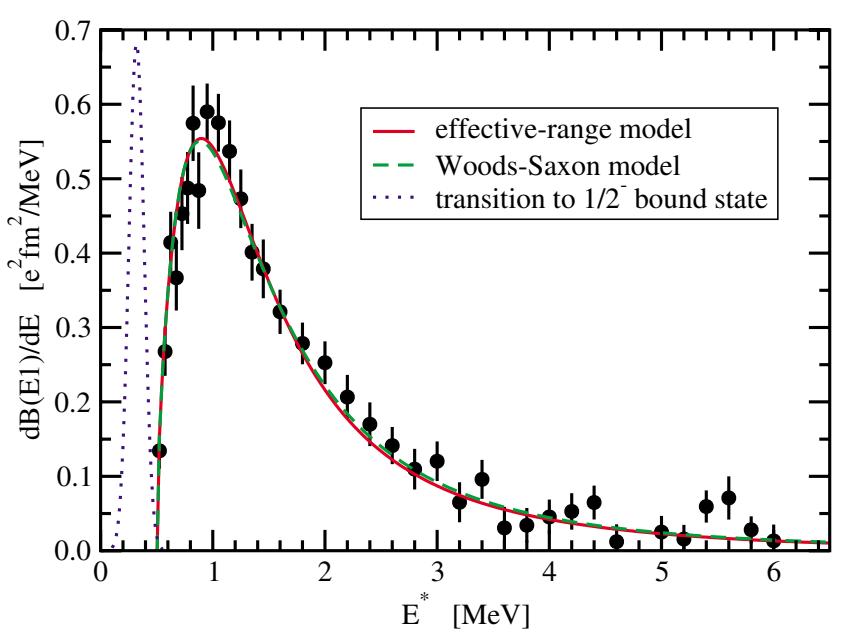

FIG. 1 (color online). Reduced transition probability as a function of the excitation energy $E^{*}=E+S_{n}$ compared to experimental data extracted from the Coulomb breakup of ${ }^{11} \mathrm{Be}[6]$.

(solid line) is compared to the experimental strength distribution [6], including corrections due to the detector response.

The obtained ANC $C_{0}=0.724(8) \mathrm{fm}^{-1 / 2}$ can be translated into a spectroscopic factor $C^{2} S$ by comparing to the ANC of a normalized single-particle wave function generated from a Woods-Saxon (WS) potential with a depth adjusted to the correct neutron separation energy. Assuming a radius of $R=2.78 \mathrm{fm}$ as above and a diffuseness parameter of $a=0.65 \mathrm{fm}$ we obtain $C^{2} S=$ 0.704(15) which compares well with the spectroscopic factors from experiment and theory as given in Ref. [6]. The model ground state wave function has a rms radius of $7.172 \mathrm{fm}$ that corresponds to a total strength of $B(E 1)=$ $1.645 e^{2} \mathrm{fm}^{2}$ according to (17). Applying the spectroscopic factor we obtain a total strength of $1.159(36) e^{2} \mathrm{fm}^{2}$. The experimental continuum strength [6] exhausts about 78(5)\% of the sum rule. The ground state transition probability to the first excited state is experimentally known to be $B(E 1)=0.116(12) e^{2} \mathrm{fm}^{2}$ [19] corresponding to $10(1) \%$ of the sum rule. This bound-state transition strength is indicated in Fig. 1 as a dotted line assuming an arbitrary FWHM of $0.160 \mathrm{MeV}$. The remaining 12(6)\% can be attributed to the $p_{3 / 2}$ state that is occupied. Comparing the experimental transition strength to results of a plane-wave calculation would underestimate the extracted spectroscopic factor.

Our ER approach can be compared to an exact calculation of the matrix elements with wave functions obtained from WS potentials. We assume the same shape of the potential with $R$ and $a$ as given above. Repeating the $\chi^{2}$ fit by adjusting the depth of the potential in the two $p$ waves independently we find $C^{2} S=0.696(15)$ close to the result of our ER approach. The exact calculation (dashed line in Fig. 1) almost coincides with our ER approximation. We can also extract the dimensionless quantities $c_{1}^{j}$ according to Eq. (9). Their modulus decreases with relative energy (much more strongly for $c_{1}^{1 / 2}$ than for $c_{1}^{3 / 2}$ ). Since the $c_{1}^{j}$ in our ER approach are constant they can be considered as an average of the actual values over the peak of the strength distribution. Taking this into account a reasonable agreement of the two approaches is found again (Table I).

In conclusion we find that low-lying electric multipole strength in one-neutron-halo nuclei can be described effectively by a few low-energy constants: the binding energy, the scattering length, and the asymptotic normalization constant. We apply our ER approach to the halo nucleus ${ }^{11} \mathrm{Be}$ and find remarkable agreement with an exact calculation. The exhaustion of the strength due to the bound state affects the extraction of the ANC and of the spectroscopic factor. In our approach there is no need to determine parameters of optical potentials from elastic scattering.

The present theoretical approach will be applied to other halo nuclei. It will be extended to higher multipolarities and to proton halo nuclei in a forthcoming publication [20]. An effective-range theory of two-neutron-halo nuclei like ${ }^{11} \mathrm{Li}$ would be very interesting but is certainly much more complex [4]. An early work related to this problem is [21].

We are grateful to T. Aumann and H. Emling for useful discussions.

[1] A. Leistenschneider et al., Phys. Rev. Lett. 86, 5442 (2001).

[2] B. Jonson, Phys. Rep. 389, 1 (2004).

[3] P. G. Hansen and B. Jonson, Europhys. Lett. 4, 409 (1987).

[4] A. Jensen et al., Rev. Mod. Phys. 76, 215 (2004).

[5] T. Nakamura et al., Phys. Lett. B 331, 296 (1994).

[6] R. Palit et al., Phys. Rev. C 68, 034318 (2003).

[7] T. Nakamura et al., Phys. Rev. Lett. 83, 1112 (1999).

[8] G. Baur, K. Hencken, and D. Trautmann, Prog. Part. Nucl. Phys. 51, 487 (2003).

[9] S. Typel and G. Baur, Phys. Rev. C 64, 024601 (2001).

[10] C. Bertulani, H.-W. Hammer, and U. van Kolck, Nucl. Phys. A712, 37 (2002).

[11] M. C. Birse et al., Phys. Lett. B 464, 169 (1999).

[12] A. M. Mukhamedzhanov and F. M. Nunes, Nucl. Phys. A708, 437 (2002).

[13] D. Baye, Phys. Rev. C 70, 015801 (2004).

[14] H. Esbensen, G. F. Bertsch, and K. Hencken, Phys. Rev. C 56, 3054 (1997).

[15] D. M. Kalassa and G. Baur, J. Phys. G 22, 115 (1996).

[16] A. Mengoni, T. Otsuka, and M. Ishihara, Phys. Rev. C 52, R2334 (1995).

[17] T. Otsuka et al., Phys. Rev. C 49, R2289 (1994).

[18] J. M. Blatt and V.E. Weisskopf, Theoretical Nuclear Physics (Springer-Verlag, New York, 1979).

[19] T. Glasmacher, Annu. Rev. Nucl. Part. Sci. 48, 1 (1998).

[20] S. Typel and G. Baur (to be published).

[21] A. B. Migdal, Sov. J. Nucl. Phys. 16, 238 (1973). 